\title{
Statistical and Domain Analytics for Informed Study Protocols
}

\author{
Nicholas R. Wheeler, Laura S. Bruckman, Junheng Ma, Ethan Wang, Carl K. Wang, Ivan Chou, Jiayang Sun \\ and Roger H. French, Member, IEEE,
}

\begin{abstract}
To optimize and extend the lifetime of photovoltaic (PV) modules, a better understanding of the modes and rates of their degradation is necessary. Lifetime and degradation science (L\&DS) is used to better understand degradation modes, mechanisms and rates of materials, components and systems in order to predict lifetime of PV modules. Statistical analytic methods were used to investigate the relationships between various subsystem characteristics related to suspected degradation pathways, as well as their impact on changes in module performance. A PV module lifetime and degradation science (PVM L\&DS) model developed in this way is an essential component to predict lifetime and mitigate degradation of PV modules. Previously published accelerated testing data from Underwriter Labs, featuring measurements taken on 18 modules with fluoropolymer, polyester and EVA (FPE) backsheets, were used to develop the analytical methodology. To populate this dataset, three performance characteristics for each module were tracked over a maximum of $\mathbf{4 0 0 0}$ hours while the modules were exposed to stressful conditions. Two of the eighteen modules' performance characteristics were measured with no exposure to stress, and then dissassembled immediately to provide baseline measurements. Eight of the sixteen remaining modules were exposed to $85 \%$ relative humidity at $85^{\circ} \mathrm{C}$ (Damp Heat, DH) and the final eight were exposed to $80 \mathrm{~W} / \mathrm{m}^{2}$ of ultraviolet light at $280-400 \mathrm{~nm}$ wavelengths and $60^{\circ} \mathrm{C}$ (UV). Four of the sixteen modules being exposed (two from $\mathrm{DH}$ conditions and two from UV conditions) were removed at each 1000 hour time point and disassembled to provide observations for eleven component level experiments, six directly related to degradation mechanisms and five to material performance characteristics. The resulting dataset comprised of coincident observations of 15 variables (time, three system-level performance variables, and eleven component-level variables) was statistically analyzed using the developed methodology. Limitations in the quantity of coincident observations constrained the statistical study to require the use of domain knowledge to pre-select a subset of variables for analysis, which introduced undesirable bias and prevented the full development of a prognostic model from this dataset alone. The results and lessons learned help guide the experimental design for better structuring further accelerated and real-world experiments, providing necessary insight in order to sample data effectively and efficiently, obtain maximum information for identifying statistically significant relationships between variables, and develop a PVM L\&DS model construction methodology to determine degradation modes and pathways present in modules and their effects on module performance over lifetime.
\end{abstract}

Index Terms-photovoltaics, statistical modeling, lifetime and degradation science, structural equation modeling.

L.S. Bruckman, N.R. Wheeler and R.H. French are with the Department of Material Science and Engineering, Case Western Reserve University, Cleveland OH, 44106 USA e-mail: (roger.french@case.edu).

J. Ma and J. Sun are with Department of Epidemiology and Biostatistics, Case Western Reserve University

E. Wang and C.K. Wang are with Underwriters Laboratories.

Ivan Chou is with Delsolar Co.

\section{INTRODUCTION}

A Recent U.S. Department of Energy workshop on Science for Energy Technologies [1] identified photovoltaics (PV) lifetime and degradation science (L\&DS) [2], [3], [4] as a critical scientific challenge for robust adoption of PV. The PVQA Task Force was developed as an international task force to work towards defining what is needed for lifetime qualification standards and tests [5]. Developing and defining useful lifetime qualification standards and tests is complicated because even single degradation modes, mechanisms and rates are not clearly understood and two-factor effects are even more complex. Therefore, a statistical and methodical approach is necessary to cross-correlate stressors, degrees of stress and degradation modes, mechanisms and rates for materials, components and systems. This cross-correlation can help provide a better understanding of degradation and lifetime performance in order to guarantee the minimal 25 year lifetime performance of PV modules [6].

\section{A. Lifetime and Degradation Science Approach}

The original method to determine the reliability of a system or component was to collect failure data and use a constant failure rate to account for product quality and environmental conditions. However, this approach is inaccurate at determining field failure rates and does not produce reliable lifetime predictions [7], [8]. In contrast, the physics of failure approach, based on failure modes and mechanisms as a function of the stress conditions, provides useful reliability information. The prognostics and health management approach assesses the reliability under application conditions and over lifetime. The combination of the physics of failure and prognostic approaches allows for continuous improvement of lifetime prediction [7], [8].

Lifetime approaches that consider system response, levels of response and degradation rates under stress conditions that include single, multi-factor, constant and cyclic conditions which vary by region are necessary. This lifetime approach must also cross-correlate real-world and accelerated lab based exposures [9], [10], [11]. An unbiased analytical approach that considers full system level modeling and a comprehensive degradation model, PV module lifetime and degradation science model (PVM L\&DS), that is refined iteratively by updated analysis is necessary in order to determine lifetime of current and new technologies.

A stress and response $(R(S))$ framework links stresses to observed responses, subsequent degradation and damage 
accumulation over the lifetime of PV materials, components and systems. Stressors can be characterized in terms of instantaneous stress level $(\sigma)$ and net or integrated stress $(S)$. A material's response $(R)$ to both $\sigma$ and $S$ can be described in Equation 1 as:

$$
R=f\left({ }^{\sigma} S\right)=\int f(\sigma) \sigma d t
$$

Degradation modes of materials can be elucidated by performing accelerated studies and a cross-correlation function can be used to predict the response observed in a system exposed to accelerated stressors compared to real-world conditions. The combination of single and multi-factor tests can lead to a better understanding of the synergistic effects of stress in a real-world environment. Response is therefore a function of the convolution of multiple stressors at their service-use conditions. Murray et al. exposed two grades of poly (methyl methacrylate) under two stress levels of full spectrum and ultraviolet light: a single-factor accelerated test. A constant "acceleration" factor was not observed for key degradation modes for the two stress conditions, demonstrating that the traditional search for a single acceleration factor is unreasonable. The $R(S)$ framework is a more versatile approach to encompass multiple stressors, stress levels and responses [9]. Mitigating key degradation modes and lifetime penalties can increase a PV module's lifetime performance.

\section{B. Statistical and Domain Analytics: L\&DS Analytics}

A PVM L\&DS model can provide knowledge of rankordered degradation modes for different climatic zones and stress conditions, which can highlight modes contributing to performance and lifetime for a particular use condition. A statistically valid approach is necessary for the development of a reliable PVM L\&DS model for accurate lifetime prediction. The statistical analysis requires a large data set in terms of variables and sample sizes, both for groups of measurements at single time points and total number of measured time points over the entire timeframe of the study as well. Statistically significant relationships (SSRs) need to be elucidated between different stressors and degradation modes and pathways. Statistics such as R-Squared Values and P-Values can be used to assess the strength of these relationships between variables quantifying degradation and performance loss. Errors such as random, measurement, systematic and method errors should be incorporated into the methodology to remove bias from the resulting model [12].

After statistical analysis is performed, domain knowledge is used to understand the correlations indicated and other results of the statistical analysis. Observations can be framed in terms of known mechanisms and pathways as well as a particular system response. Domain knowledge can be used to identify the probable active and quiescent pathways. Many different pathways can be activated by different stressors or stress levels and need to be incorporated into the PVM L\&DS model. Figure 1 shows some example PV module degradation pathways, which are based on observations from both realworld exposed modules and modules subjected to accelerated

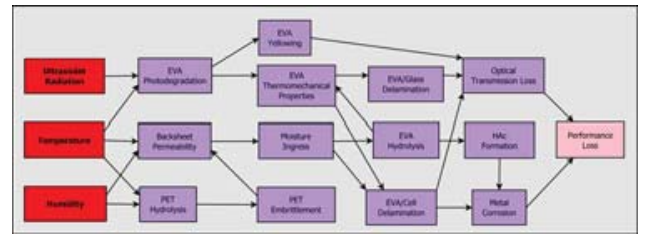

Fig. 1. Example theoretical PV module performance degradation pathways

test conditions [13], [14], [15], [16], [17], [18]. When module failure modes can be attributed to processes that are only activated under accelerated exposure conditions or accelerated exposure conditions are chosen that fail to activate pathways observed in field-exposed modules, it signals inappropriate exposure conditions with little basis in reality. To assess which testing conditions recreate sensible real-world module degradation modes for the prediction of module performance, the deciding factor will be which degradation pathways are activated by the chosen stress levels using the stress and response framework and lifetime and degradation science [9].

The iterative nature of the PVM L\&DS model allows for the continuous refinement of the model. As more knowledge is gained through accelerated exposure studies and real-world data and as new technologies are incorporated into the model, the correlations and predictions will become stronger over time; therefore producing a predictive, statistically significant prognostic model. Proper documentation of the limitations and scope of a predictive model is important to ensure that a model is providing useful information on lifetime [19].

\section{UL PREVIOUS RESULTS}

The data used for the statistical modeling was published by E. Wang et. al. [20]. Eighteen commercially available polycrystalline 60-cell solar PV modules made with FPE backsheets were fabricated under identical processing conditions by DelSolar [21]. Eight PV modules were subjected to damp heat $(\mathrm{DH})$ aging, eight modules were exposed to UV and two modules were used as control samples and not exposed. Test laminates of ethylene-vinyl acetate (EVA) and glass were fabricated based on the module design and lamination process and were used for interfacial adhesion tests. The edge of these test laminates were sealed with a waterproof and temperature resistance acrylic foam tape to avoid excess moisture ingress.

There were no specified variations in the PV modules used for the experiments and the intention of the work was not to analyze differences in results between constrasting PV modules. By keeping the choice of PV module a constant and varying the exposure conditions rather than comparing performances between multiple module types, a wider range of degradation modes can be observed and characterized within a single PV module system. The scope of the data collected for each variable under each exposure type over time can be seen in Figure 2.

\section{A. Damp Heat Exposure}

Damp heat exposure entailed $85^{\circ} \mathrm{C}$ ambient temperature and $85 \%$ relative humidity and is described in test 10.13 of IEC 
61215 Ed.2. [22]. The TPE PV modules and test laminates were aged in damp heat conditions four times longer than the IEC standard of 1000 hours. Two of the original eight modules were removed from the chamber at each 1000-hour time increment and destructively disassembled, and packaging materials were collected and tested, until a maximum exposure time of 4000 hours [20].

\section{B. UV Irradiance Exposure}

The UV exposure was similar to test 10.10 of IEC 61215 Ed.2, [22] but with higher light intensity, approximately 80 $\mathrm{W} / \mathrm{m}^{2} \mathrm{UV}$ irradiance at $280-400 \mathrm{~nm}$ wavelengths plus an additional $15 \%$ of the total irradiance at the backside of the PVMs and test laminates. The module temperature was controlled at $60^{\circ} \mathrm{C}$, and the relative humidity was uncontrolled. The total irradiance, until 4000 hours, was about 20 times higher than IEC standard of $15 \mathrm{kWh} / \mathrm{m}^{2}$. Two of the original eight modules were removed from the chamber at each 1000-hour time increment and destructively disassembled, and packaging materials were collected and tested, until a maximum exposure time of 3000 hours [20].

\section{PV Module Responses}

Experiments were performed on the module, component and mechanistic level for modules from each of the exposure conditions in order to extract information on each of these levels. This provides a more in-depth study of the degradation modes and how these modes affect components and the overall module performance. In addition, performing the same experiments for both damp heat and UV exposures leads to an understanding of which degradation modes and pathways are active during each type of stress. The results of these experiments are directly related to time of exposure since conditions were held constant in the damp heat and UV exposures.

\section{System Responses}

Three system responses were measured for each of the 16 PV modules which included peak power (Pmax), fill factor (FF) and wet insulation resistance (WetIns). The system-level responses were measured every 500 hours for both the damp heat and UV experiments from 0 to 2500 hours, and then every 250 hours from 2500 to 4000 hours for damp heat and 2500 to 4250 hours for UV experiments [20]. Pmax is the peak power of a photovoltaic module, and is determined by measuring current and voltage while varying resistance under defined illumination. The power reduction is driven by reduced FF (a metric describing the ideality of the shape of the current and voltage curve) and increased series resistance. These reductions are initially interpreted as corrosion taking place at the electrical interconnects [23], [24], [25]. Wet insulation resistance testing according to IEC 61215 [22] is intended to verify that a PV module's packaging materials have sufficient electrical insulation properties to reduce the possibility of fire and electric hazards, even when the module is wet.

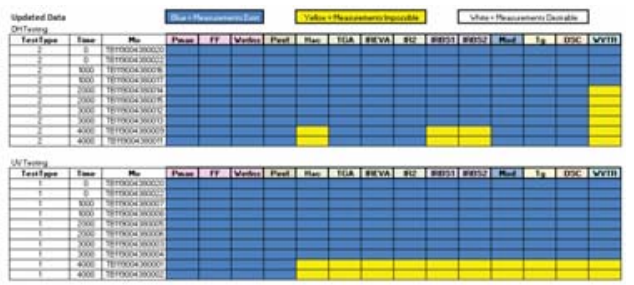

Fig. 2. Summary of data from PV modules for each of the system and unit data for the DH (top) and the UV (bottom) experiments. The blue squares indicates data that was taken for the time point and the yellow squares indicate data that was not collected [20].

\section{E. Unit Experiments}

Ten component-level experiments were performed on the modules and one unit experiment was performed on the test laminates. These experiments can be separated into two categories: performance (peel strength of the EVA-glass interface in the test laminates (Peel), EVA modulus (Mod), EVA glass transition temperature $\left(\mathrm{T}_{g}\right)$, water vapor transmission rate of the backsheet (WTVR) and differential scanning calorimetry of EVA (DSC)) and mechanistic (two IR measurements of EVA hydrolysis IREVA and IR2, thermogravimetric analysis of EVA (TGA), EVA acetic acid content (Hac), and 2 IR measurements of backsheet PET hydrolysis IRBS1 and IRBS2). The unit experiments were performed on component materials and subsystems of dissassembled modules every 1000 hours of testing [20].

\section{L\&DS ANALYTICS: METHODS}

1) Data Determination: Two unexposed modules were disassembled and unit-level variable measurements taken to capture a shared baseline between the DH and UV exposures. Two additional modules per stress condition were disassembled at each 1000-hour interval (up to 4000 hours for the DH exposure and 3000 hours for the UV exposure) for measurements of the unit-level variables. Although the system-level variables (Pmax, FF, WetIns) were measured more frequently than 1000hour intervals, they share only these time points with the unitlevel variable measurements. Only these shared time points allow comparison between measurements of different variables from identical modules, which is the basis of the coincidence of observations necessary for this statistical analysis.

The number of measurements at each time point for each variable was not consistent between different variables. Some of the variables were measured multiple times (2-6 per harvested module at each time point), while others were only measured once. Additionally, individual measurements at identical time points between different variables are only comparable to one another on the basis of their sample origin (which module they came from); they do not correspond directly to one another even if the number of measurements is identical. Therefore, in the event of multiple measurements of a single variable from a single module at a single time point, the measurements were averaged to a single value to enable comparison between variables.

Variables differ slightly in regards to how the results of the measurements were interpreted into single discrete values. 
The Pmax, FF, WetIns, WVTR, Hac, Peel and Tg measurements each provide a single value which can be used without modification. The test results of IREVA, IR2, IRBS1 and IRBS2 are absorption spectra, which are interpreted into specific meaningful ratios of integrated absorption bands to provide singular values. The TGA test provides a curve of sample weight versus temperature, portions of which (characterized by changes in the slope of the curve) represent losses of specific chemical groups. The total weight lost over a particular interval corresponds to the amount of acetate side groups present in the polymer, which functions as a single discrete value for each TGA curve. The DSC test provides a curve describing the change of heat flow into a sample over a scanned temperature range. Changes in the heat flow correspond to changes in the degree of crystallinity present in the sample. The degree of crystallinity can be quantified, which functions as a single discrete value for each DSC curve. Dynamic mechanical analysis results (Mod) in a curve describing changes in material modulus over a scanned temperature range. A single temperature was chosen $\left(71.363^{\circ} \mathrm{C}\right)$ and the modulus at that point used to describe each dynamic mechanical analysis curve as a single discrete value.

2) Variable Selections: In a typical data analysis problem, there are predictors and response variables. Often, some predictors have little or no influence on the response variable. The procedure to identify those predictors or to select the "best" subset of the predictors, such that the statistical model based on this subset of predictors is as significant as the full model based on all the predictors, is called variable selection. This data set features small $\mathrm{n}$ and large $\mathrm{p}$ data (meaning a small number of observations for a comparatively large number of variables) although this is not the typical case. The number of variables that can be considered in the statistical analysis is restricted by the number of observations per variable according to the formula $n-2=v$, where $n$ represents the number of observations and $v$ represents the number of variables that can be included in the analysis. In this case $n=8$ observations, so only 6 variables can be included. This means more variables exist than can be simultaneously included into the modeling, and so variables must be chosen on the basis of measurement confidence and domain knowledge based expectation of significance to the overall model. Due to the limited amount of data, domain knowledge has been used to select contrasting subsets of predictors to use for the initial variable selection step of constructing the statistical pathway models.

\section{A. Mapping to Domain Experience}

The models relating the variables should be checked for consistency with domain knowledge about the phenomena being measured. With respect to time, chemical and physical processes are typically either linear or exponential, so if a polynomial model is found to provide the best fit, this could indicate that there are change points resulting from the influence of combined effects. Additionally, phenomena anticipated to be exponential could appear linear if test conditions are not aggressive enough to elicit sufficient response over the
TABLE I

VARIABLES INCLUDED IN THE MODEL DEVELOPMENT BASED ON DOMAIN KNOWLEDGE. TIME IS INCLUDED FOR EACH OF THE MODELS AS THE ONLY WHOLLY INDEPENDENT VARIABLE.

\begin{tabular}{|c|c|}
\hline $\begin{array}{c}\text { System Level } \\
\text { Response }\end{array}$ & Unit Level Variables \\
\hline Pmax or FF & Hac or TGA, IREVA or IR2, IRBS1 or IRBS2 \\
\hline
\end{tabular}

total measurement time. Domain knowledge should always be applied to the results of the statistical analysis; relationships indicated statistically that violate domain knowledge based expectations, or domain knowledge based expectations that are not reflected by the statistical analysis, both indicate that further investigation is required.

\section{B. Statistical Modeling}

Presently two principles have been used for investigating relationships between the chosen initial subsets of variables. The first principle examines only univariate relationships based on a selection of known functional forms directly from one variable to another, and performs iterative variable selection steps on the basis of a 0.2 cutoff of adjusted-r-squared value. The second principle examines the collective additive influence of variables upon one another utilizing Akaike Information Criterion (AIC) values to perform stepwise variable selections iteratively to indicate networks of relationships between the variables [27].

The development of the PVM L\&DS model allows for the refinement of the model with more data over time; therefore, with each iteration of the modeling procedures in light of additional data, increasingly accurate statistical results will be obtained.

\section{L\&DS ANALYTICS: RESULTS}

\section{A. Variables}

For both DH and UV, the system-level responses examined were Pmax and FF. Mechanistic unit-level predictors were chosen for the initial subset of predictors because they could be attributed to specific, known phenomena. Based upon domain knowledge and measurement confidence, in addition to time the chosen variables included in the model are listed above. (Table I )

\section{B. Statistical Models}

All 32 possible combinations of the chosen variables were modeled with each of the two previously described principles of variable selection. The resulting networks of statistically significant relationships were informative and mapped well to domain knowledge [27]. A full prognostic model was not developed due to some limitations in the data set, though much was learned about how to structure future studies to be more amenable to the developed statistical analysis approach. 


\section{L\&DS ANALYTICS: DiscUSSIONS}

\section{A. Insights about Data}

The data used in this analysis were sometimes averaged; however, it is preferable to use all the data available whenever possible. As an example, aspects of a collection of values such as their standard deviation can be carried into statistical analysis along with their mean value, when the values cannot be compared on an individual basis as seen in this study. Further iterations of the statistical analytics for developing the PVM L\&DS model will use all possible data points for a more statistically accurate model development.

Important information can be understood from this data set about accelerated experiment design for statistically sound practice. It is critically necessary to have a sufficient amount of sampling at numerous enough time points throughout the experiment. In the case of this data set, having time points at every five hundred hours allows for better model fits to the data for the system level variables. Also, change points are more readily apparent when data is collected more frequently because the change between the linear and exponential responses can be more precisely identified.

The number of measurements taken for each experiment is very important for modeling and understanding statistically significant relationships between the variables. The number of observations needs to be large enough to be able to include every variable, which ultimately allows for the relationships between the system level responses and the unit experiments to be elucidated. In the case of this data, the number of observations was small which necessitated the use of domain knowledge to determine which variables should be used in the modelings of statistically significant relationships. Due to this, a completely unbiased approach was not possible to explore the data without influencing the outcome beforehand by constraining the possibilities.

\section{B. Unbiased Analysis}

It is necessary to do the analysis with a full set of all measured variables to avoid prebiasing the statistical model development. In the case of our results, the number of observations was inadequate for the number of variables, so it was necessary to choose between the variables using domain knowledge. A study can be structured so that the number of observations is large compared to the number of variables, enabling all the variables to be included during model development. To do this, one can increase the number of time points at which data is collected (e.g. increasing the entire time frame or the frequency of measurement), increase the sample size at each time point (e.g. 4 observations of a variable instead of 2) or combine both of these approaches to increase the total number of observations, and thus the maximum number of variables that can be included in the model development process. In the case of this analysis, the small data set constrained the number of includable variables and forced a pre-selection with domain knowledge prior to the statistical analysis.

\section{Statistical Analysis Framed By Domain Knowledge}

More than one variable related to a particular suspected degradation mechanism can be measured in order to fully explore single mechanistic responses from many angles. For example, measurements aiming to describe the precursors, product accumulation and reactant reduction can be used to obtain a broader range of information about the degradation pathway that yields acetic acid. Though these various measurements may seem redundant, they are actually very useful for increasing confidence in the observed results, and identifying differences in the efficacy of certain kinds of measurements as compared to one another.

\section{Linear \& Exponential Responses for Chemical/Physical Processes}

Typical chemical and physical processes display either linear or exponential progression through time. Quadratic responses aren't known to be characteristic of these processes. Therefore, if a variable transformation appears to be quadratic, it can be an indication that a change point in a process is occuring after an accumulation period. Apparent linear responses also warrant careful consideration, as even exponential responses can appear linear on a short enough timescale, or with infrequent sampling.

\section{E. Stress Levels and Total Response}

Stress levels must be sufficient to produce a measurable response within the time frame of an experiment. If the stress is too small, as in the case of the modules exposed to the UV stress conditions of this study, there is insufficient information to define and understand the relationships between measured responses and the model cannot be significantly improved. Therefore, either the stress intensity or the time frame of the entire exposure must be increased to fully realize the value of the experiments.

In the case of the damp heat experiments, the stress is extreme and causes a large amount of degradation by 4000 hours. Having more frequent data collection at the beginning of this experiment would provide more numerous insights into the degradation modes prior to the complete failure of the modules. Also, this high stress level can induce additional degradation pathways that do not reflect responses to actual use environments, and so the experiment may more appropriately recreate degradation experienced during real world operation if conducted under less harsh conditions.

The comprehensive PVM L\&DS model needs to encompass the possibility of all degradation pathways, even those not activated under an experimental stress condition being studied. In order to understand the degradation modes, mechanisms and rates to accurately predict lifetime of PV modules, all degradation pathways need to be fully explored and information about these pathways elucidated. By cross-correlating a wide variety of stress levels, the relationship between stress and module performance can be examined and understood. 


\section{CONCLUSION}

Traditional lifetime reliability methods do not provide a statistical means to accurately predict lifetime of PV modules. In order to gain insights into lifetime through accelerated testing, the information collected needs to be appropriate for sound statistical practice. Although this particular data set is not sufficient to make a complete prediction for 25-year lifetime guarantee of modules, it does provide a means by which to begin to amass the necessary data. Experiments must be structured carefully in order to balance a maximum amount of collected data alongside effective and efficient sampling to keep costs as low as possible while maximizing realized value. A large enough data set is essential in order to support good statistical knowledge. It is beneficial to have more than one test investigating the same degradation mode, as in the case of acetic acid production. Obtaining information on precursors, reactants and products allows for a broader understanding of the degradation mode's impact on the system's performance metric responses. Accelerated testing protocols should be constructed so that there is sufficient degradation occurring to be visible for measurement, while still activating responses expected to be present in real-world conditions. The activation of degradation modes by extremely harsh conditions needs to be recognized so that true degradation modes can be identified and characterized that are relevant to real use conditions.

\section{ACKNOWLEDGMENTS}

The authors acknowledge funding from Underwriter Laboratories. Research was performed at the SDLE Center at Case Western Reserve University, funded through the Ohio Third Frontier, Wright Project Program Award Tech 12-004.

\section{REFERENCES}

[1] J. Hemminger, "From quanta to the continuum: Opportunities for Mesoscale Science," A Report from the Basic Energy Sciences Advisory Committee, Tech. Rep., 2012.

[2] M. P. Murray, D. Gordon, S. A. Brown, W.-C. Lin, K. A. Shell, M. A. Schuetz, S. Fowler, J. Elman, and R. H. French, "Solar radiation durability framework applied to acrylic solar mirrors," Proc. SPIE 8112, Reliability of Photovoltaic Cells, Modules, Components, and Systems IV, 811203 (September 13, 2011), pp. $811203-811$ 203-10, 2011. [Online]. Available: +http://dx.doi.org/10.1117/12.893827

[3] R. French, M. Murray, W.-C. Lin, K. Shell, S. Brown, M. Schuetz, and R. Davis, "Solar radiation durability of materials components and systems for low concentration photovoltaic systems," in Energytech, 2011 IEEE, May 2011, pp. $1-5$.

[4] R. French, J. Rodríguez-Parada, M. Yang, M. Lemon, E. Romano, and P. Boydell, "Materials for concentrator photovoltaic systems: Optical properties and solar radiation durability," in AIP Conference Proceedings, vol. 1277, 2010, p. 127.

[5] "International PV Module Quality Assurance Task Force, established by NREL, AIST, PVTEC, U. S. DOE, EU JRC, SEMI PV Group to develop PV Lifetime Qualification Testing," 2012.

[6] J. Hemminger, G. Crabtree, and A. Malozemoff, "Science for energy technology: Strengthening the link between basic research and industry," A report from the Basic Energy Sciences Advisory Committee, US Department of Energy, 2010.

[7] M. G. Pecht, Introduction. John Wiley \& Sons, Inc., 2008, pp. 1-24. [Online]. Available: http://dx.doi.org/10.1002/9780470385845.ch1

[8] - Physics-of-Failure Approach to PHM. John Wiley \& Sons, Inc., 2008, pp. 73-84. [Online]. Available: http://dx.doi.org/10.1002/ 9780470385845.ch4
[9] M. P. Murray, L. S. Bruckman, and R. H. French, "Photodegradation in a stress and response framework: Poly(methyl methacrylate) for solar mirrors and lens," Journal of Photonics for Energy, vol. 2, no. 1, pp. 022 004-022004, 2012. [Online]. Available: +http://dx.doi.org/10.1117/1.JPE.2.022004

[10] M. Murray, L. Bruckman, and R. French, "Durability of acrylic: Stress and response characterization of materials for photovoltaics," in Energytech, 2012 IEEE, May 2012, pp. 1 -6.

[11] L. Bruckman, M. Murray, S. Richardson, S. Brown, M. Schuetz, and R. French, "Degradation of back surface acrylic mirrors: Implications for low concentration and mirror augmented photovoltaics," in Energytech, 2012 IEEE, May 2012, pp. 1-4.

[12] R. Bagozzi and Y. Yi, "Specification, evaluation, and interpretation of structural equation models," Journal of the Academy of Marketing Science, vol. 40, no. 1, pp. 8-34, 2012. [Online]. Available: http://dx.doi.org/10.1007/s11747-011-0278-x

[13] F. Pern, "Factors that affect the EVA encapsulant discoloration rate upon accelerated exposure," in Photovoltaic Energy Conversion, 1994., Conference Record of the Twenty Fourth. IEEE Photovoltaic Specialists Conference - 1994, 1994 IEEE First World Conference on, vol. 1, Dec 1994, pp. $897-900$ vol.1.

[14] F. Pern and S. Glick, "Photothermal stability of encapsulated Si solar cells and encapsulation materials upon accelerated exposures," Solar Energy Materials and Solar Cells, vol. 61, no. 2, pp. 153-188, 2000.

[15] D. King, W. Boyson, and J. Kratochvill, "Photovoltaic array performance model. Sandia National Laboratories, Albuquerque, New Mexico, 87185-0752," 2004.

[16] D. L. King, M. A. Quintana, J. A. Kratochvil, D. E. Ellibee, and B. R. Hansen, "Photovoltaic module performance and durability following long-term field exposure," Progress in Photovoltaics: Research and Applications, vol. 8, no. 2, pp. 241-256, 2000. [Online]. Available: http://dx.doi.org/10.1002/(SICI) 1099-159X(200003/04)8:2〈241::AID-PIP290〉3.0.CO;2-D

[17] W. Gambogi, "Comparative performance of backsheets for photovoltaic modules," in 25th European Photovoltaic Solar Energy Conference and Exhibition (EU PVSEC), 2010.

[18] J. Del Cueto and T. McMahon, "Analysis of leakage currents in photovoltaic modules under high-voltage bias in the field," Progress in Photovoltaics: Research and Applications, vol. 10, no. 1, pp. 15-28, 2002.

[19] J. Elerath and M. Pecht, "IEEE 1413: A standard for reliability predictions," Reliability, IEEE Transactions on, vol. 61, no. 1, pp. 125 -129, March 2012.

[20] E. Wang, H. E. Yang, J. Yen, S. Chi, and C. Wang, "Failure modes of evaluation of pv module via materials degradation approach," in $P V$ Asia Pacific Expor and Conference, 2012. Elsevier Science Direct, in press.

[21] DelSolar Co., Ltd. Headquarters 6 Kebei $2^{\text {nd }}$ Road, Shunan Science Park, Zhunan Township, Miaoli Country 35053, Taiwan, R. O. C.

[22] IEC 61215, "IEC Standard 61215 second edition-Crystalline silicon terrestrial photovoltaic (PV) modules-design qualification and type approval," ASTM International, 2005, IEC 61215:2005(E).

[23] B. Ketola and A. Norris, "Degradation mechanism investigation of extended damp heat aged PV module," in 26th EUPVSEC, 2011.

[24] K. Whitfield, A. Salomon, S. Yang, and I. Suez, "Damp heat versus field reliability for crystalline silicon," in Photovoltaic Specialists Conference (PVSC), 2012 38th IEEE, June 2012, pp. 001864 -001 870.

[25] S. Sakamoto, T. Kobayashi, and S. Nonomura, "Epidemiological analysis of degradation in silicon photovoltaic modules," Japanese Journal of Applied Physics, vol. 51, p. 10NF03, 2012. [Online]. Available: http://jjap.jsap.jp/link?JJAP/51/10NF03/

[26] $\mathrm{R}$ Development Core Team, $R: A$ Language and Environment for Statistical Computing, R Foundation for Statistical Computing, Vienna, Austria, 2008, ISBN 3-900051-07-0. [Online]. Available: http://www.R-project.org

[27] L. S. Bruckman, N. R. Wheeler, J. Ma, E. Wang, C. K. Wang, I. Chou, J. Sun, and R. H. French, "Statistical and domain analytics applied to PV module lifetime and degradation science," IEEE Open Access, Invited Paper, in press. 


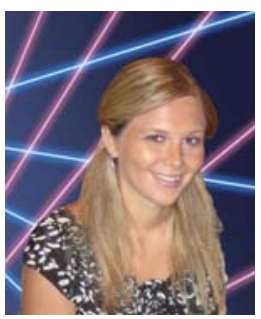

Laura S. Bruckman Dr. Laura S. Bruckman is a research associate at CWRU. Dr. Bruckman received her $\mathrm{Ph} . \mathrm{D}$. in analytical chemistry from the University of South Carolina in Dr. Michael L. Myrick's research group in August 2011. Her research while at University of South Carolina involved developing method for the rapid classification of phytoplankton cells using single-cell fluorescence measurements, optical trapping and multivariate optical computing. Dr. Bruckman's work since joining Dr. Roger French's group at CWRU has been on the degradation mechanisms of acrylic and acrylic back surface mirrors in order to better understand the Lifetime and Degradation Science of these materials.

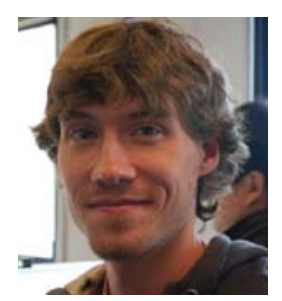

Nicholas R. Wheeler Nicholas Wheeler is a $\mathrm{PhD}$ student researching as part of Dr. French's Solar Durability and Lifetime Extension Center at CWRU. He received his M.S. in Macromolecular Science and Engineering from Case Western Reserve University in Dr. Korley's research group in 2011, and joined Dr. French's research group in April 2012. Nicholas's work at the SDLE Center involves developing prognostic models for PV module degradation under real time and accelerated stress exposures, subsystems such as backsheet assemblies. with special focus towards the role of polymeric

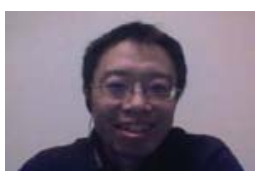

Jungheng Ma Dr. Junheng Ma received his Ph.D. in Statistics from Case Western Reserve University. His research interest includes clustering and classification, Bayesian inference, sampling techniques, and sample size determination.

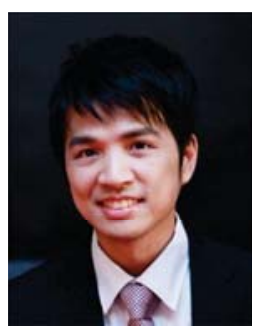

Ethan Wang Ethan Wang received his M.S. in Chemistry from National Tsing Hau University, Taiwan in 2002. He joined Underwriters Laboratories Corporate Research team in 2005 as a research scientist. Ethans experience has focused in aging and characterization of polymeric materials. Since 2007, he has conducted researches related to reliability of PV modules and PV materials. His work involves acceleration aging on PV grounding connectors, characterization of PV encapsulation and backsheets, PV failure modes analysis, as well as outdoor weathering of PV and CPV modules.

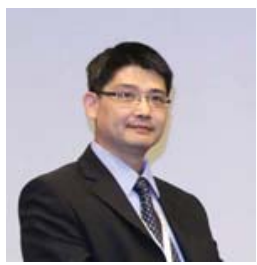

Carl K. Wang Carl K. Wang is a Research Manager of Corporate Research at Underwriters Laboratories Taiwan Co., Ltd., responsible for managing UL Corporate Research activities in Taiwan. This team focuses on materials science research for the safety aspects of various applications such as Battery, BioFuel, Photovoltaics, Wire \& Cable, PCB, etc.

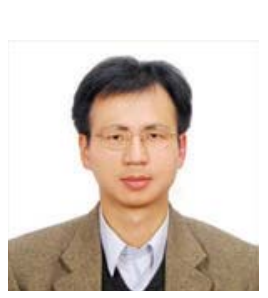

Ivan Chou Ivan Chou received his M.S. in Applied Chemistry from National Chiao Tung University, Taiwan in 1992. He joined Delsolar Co., Ltd. in 2010 as senior manager of quality engineer department. Before worked in PV industry, Ivans experience was in flat panel display and has concentrated on the process integration and materials analysis of TFT LCD and OLED. Since 2010, he has conducted activities related to failure analysis and reliability evaluation of PV modules. His work involves PV failure modes identification, outdoor weathering of PV modules and lifetime prediction model development.

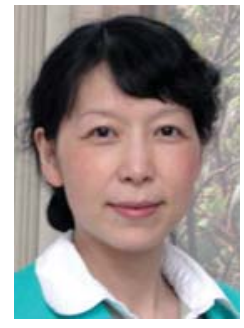

Jiayang Sun Dr. Jiayang Sun is Professor of Statistics, and Director of SR2c, Staitistical Research, Computing and Collaboration, in the Department of Epidemiology and Biostatistics at Case Western Reserve University. She received her Ph.D. in Statistics from Stanford University in 1989. She is an elected Fellow of American Statistical Association (ASA), an elected Fellow of Institute of Mathematical Statistics (IMS), and an elected Member of International Statistical Institute (ISI). She has served on various editorial boards and committees in the ASA, IMS, and other national and international professional panels. Suns research is broadly on statistical methodology, theory and computing, notabally in simultaneous inference and multiple testing; biased sampling and measurement error problems; mixtures and image analysis; bioinformatics, data mining, and large, high-dimensional data analysis; statistical computing and graphical methods; semiparametrics and random fields. Her interdisciplinary work includes those in astronomy, computer science, enviroment, imaging, material sceince, neuroscience, and other medical sciences.

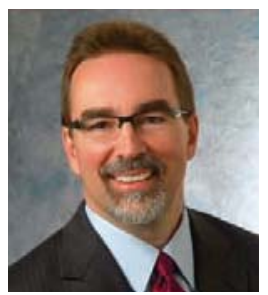

Roger H. French Dr. Roger French (F. Alex Nason Professor, Department of Materials Science and Engineering) joined CWRU in August 2010 after 24 years of conducting basic research and product development in DuPont's Central Research. He is the director of the Solar Durability and Lifetime Extension Center at CWRU. Dr. French has a broad experience in developing and commercializing optical materials for many different applications and in optimizing these materials for improved radiation durability and lifetime. Dr. French is also a nationally recognized expert in Lifetime and Degradation Science (L\&DS) for commercial applications, evidenced by his work on attenuating phase shift photomasks, fluoropolymer pellicles for photolithography, immersion lithography imaging fluids, and materials for concentrating photovoltaic systems. He has 22 issued patents and more than 145 publications. 